\title{
Philosophiques
}

Jean Grondin et Garth Green, dir., Religion et vérité. La philosophie de la religion à l'âge séculier, Strasbourg, Presses Universitaires de Strasbourg, coll. « Philosophie de la religion », 2017, 282 pages

\section{Giulio Mellana}

Volume 45, numéro 1, printemps 2018

URI : https://id.erudit.org/iderudit/1048632ar

DOI : https://doi.org/10.7202/1048632ar

Aller au sommaire du numéro

Éditeur(s)

Société de philosophie du Québec

ISSN

0316-2923 (imprimé)

1492-1391 (numérique)

Découvrir la revue

Citer ce compte rendu

Mellana, G. (2018). Compte rendu de [Jean Grondin et Garth Green, dir., Religion et vérité. La philosophie de la religion à l'âge séculier, Strasbourg,

Presses Universitaires de Strasbourg, coll. « Philosophie de la religion », 2017, 282 pages]. Philosophiques, 45(1), 310-316. https://doi.org/10.7202/1048632ar d'utilisation que vous pouvez consulter en ligne. 
Jean Grondin et Garth Green, dir., Religion et vérité. La philosophie de la religion à l'âge séculier, Strasbourg, Presses Universitaires de Strasbourg, coll. «Philosophie de la religion», 2017, 282 pages.

Actes du Colloque 20 I 5 de la Société francophone de la philosophie de la religion, Religion et vérité est un ouvrage qui se propose d'étudier et d'approfondir le sens de la vérité religieuse dans l'époque qui est la nôtre, fortement marquée par ce que les historiens appellent "sécularisation».

C'est pourquoi, dès l'introduction ${ }^{1}$, Jean Grondin place cet ouvrage dans le tracé de L'âge séculier de Charles Taylor ${ }^{2}$, dont la voix ouvre le volume avec la transcription d'une discussion publique ayant eu lieu le 2 octobre 20I 5 , à l'Université McGill, entre Charles Taylor, Jean Grondin et quelques intervenants ${ }^{3}$. Taylor précise alors que l'expression «âge postséculier» (Habermas) est à son avis susceptible d'une mécompréhension, car elle donnerait à penser qu'il existe un âge après celui de la sécularité, tandis que la post-sécularité serait à entendre plus précisément comme un changement de regard à l'intérieur du même âge séculier; un regard, donc, qui cherche à être à la hauteur de la sécularité que nous habitons.

La première section de l'ouvrage collectif, intitulée «La religion comme expérience de vérité», recueille les interventions de Jean Greisch, Jean Grondin, Ghislain Waterlot, Pierre Gisel, Carla Canullo et Yannick Courtel. Greisch approche magistralement le thème par une exégèse de l'Évangile de Jean ${ }^{4}$. Le point de départ est une analyse du "faire la vérité» (Jn 5,2I), par quoi Greisch conduit ensuite le lecteur, à travers les questions de la liberté

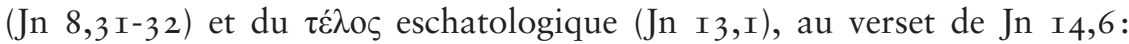
"Moi, je suis le Chemin, la Vérité et la Vie », que le philosophe luxembourgeois lit de la manière suivante: "Je suis en chemin, j'ai rendez-vous avec la vérité, je ne suis pas aussi vivant que je pourrais l'être $e^{5}$ » Le "rendez-vous avec la vérité ${ }^{6}$ » représente le cœur de la plus récente réflexion de Greisch et son noyau original, et l'engage dans une polémique tant avec le " "donjua-

1. Jean Grondin, «Introduction. Comment penser la vérité de la religion aujourd'hui ?", dans Jean Grondin et Garth Green, dir., Religion et vérité. La philosophie de la religion à l'âge séculier, Strasbourg, Presses Universitaires de Strasbourg, coll. "Philosophie de la religion", 20I7, p. 7-I 2.

2. Charles Taylor, L'âge séculier, trad. P. Sadivan, Paris, Éditions du Seuil, 20II, I339 pages.

3. "Qu'est-ce que l'âge séculier? Discussion avec Charles Taylor", dans Jean Grondin et Garth Green, dir., Religion et vérité. La philosophie de la religion à l'âge séculier, p. I3-20.

4. Jean Greisch, "Veritas semper major". La vérité dans l'horizon de l'eschatologie", dans Jean Grondin et Garth Green, dir., Religion et vérité. La philosophie de la religion à l'âge séculier, p. 23-37.

5. Ibid., p. 29.

6. Cf. Jean Greisch, Rendez-vous avec la vérité, Paris, Hermann, 20I7, 376 pages. 
nisme religieux" des touristes postmodernes ${ }^{7}$ », tant avec la soi-disant postvérité, mais aussi avec les philosophies de l'adieu à la vérité, telles la "pensée faible» de Gianni Vattimo. En conclusion, Greisch rencontre la voix de Franz Rosenzweig et sa théorie messianique de la connaissance, à qui il emprunte la notion de Bewährung, qu'il propose de traduire par "avération» au lieu de "vérification». C'est en revanche en s'appuyant sur une signification forte du terme "vérification " que Grondin tâche, par sa contribution $^{8}$, de fournir une méthode pour juger de la vérité propre à la religion. Une telle méthode doit se fonder sur trois vérifications distinctes: I) de la factualité historique, 2) de la véracité des prophéties, 3) de l'authenticité des prédications. Ensuite, le philosophe québécois montre, en s'appuyant sur l'herméneutique gadamérienne, que le propre de l'expérience de la vérité religieuse est d'être transformatrice. La transformation opérée, continue-t-il, par la vérité-révélation est en accord avec la raison, car «l'effort essentiel de la raison est de reconnaître un sens à notre expérience" ", et donc à l'expérience de cet appel à la transformation. Selon une démarche opposée à celle de Grondin et néanmoins complémentaire, le texte de Waterlot nous invite à aborder la question sub specie mysticae ${ }^{10}$. Ainsi, à la vérité d'une telle expérience appartiennent deux caractères remarquables: «I) le fait que c'est une expérience dont le fond est secret; 2) le fait que c'est une expérience "impossible" — au regard de notre intelligence ${ }^{11}$ ". Après avoir montré le propre de l'expérience (mystique) de la divinité, l'auteur peut conclure que "la vérité de l'expérience [religieuse] ne ressemble à aucune autre, mais elle demeure vérité si l'on entend par vérité l'éclat de la présence d'une réalité irréductible $^{12} »$. Si Waterlot fait appel au repliement mystique sur soi, Gisel envisage, tout en partant de la situation contemporaine occidentale manifestement multiculturelle et multireligieuse, la nécessité de s'ouvrir à l'autre dans le but de créer un "espace de débat ${ }^{13}{ }^{»}$. Il peut être utile de mettre cette contribution en parallèle avec deux autres publications du même auteur: Traiter du religieux à l'université ${ }^{14}$ et "Résistances des particularités et

7. Jean Greisch, "Veritas semper major". La vérité dans l'horizon de l'eschatologie", p. 33 .

8. Jean Grondin, "L'expérience de vérité de la religion », dans Jean Grondin et Garth Green, dir., Religion et vérité. La philosophie de la religion à l'âge séculier, p. 39-48.

9. Ibid., p. 45 .

10. Ghislain Waterlot, "La vérité de l'expérience religieuse est-elle sui generis?", dans Jean Grondin et Garth Green, dir., Religion et vérité. La philosophie de la religion à l'âge séculier, p. 49-56.

11. Ibid., p. 50.

12. Ibid., p. 56.

13. Pierre Gisel, "Pourquoi et comment reposer la question de la vérité en matières religieuses ", dans Jean Grondin et Garth Green, dir., Religion et vérité. La philosophie de la religion à l'âge séculier, p. 57-68.

14. Pierre Gisel, Traiter du religieux à l'université, Lausanne, Antipodes, 20II, I74 pages. 


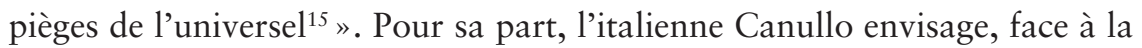
tradition moderne, une "autre voie possible» pour la conjonction de religion et vérité ${ }^{16}$. Par cette autre voie, elle approfondit étymologiquement la veritas latine (la philosophe se souvient ici de l'à $\lambda \hat{\eta} \theta \varepsilon ı \alpha$ heideggérienne) pour en découvrir des références linguistiques insoupçonnées au vouloir et au désir. Canullo interprète ce couple conceptuel en termes de libres vouloir et désir, c'est-à-dire comme "assentissement libre » ${ }^{17}$, qui donne une véritable chair à la vérité, en la soustrayant du domaine de ce qui est purement formel. La contribution de Courtel est aussi explicitement liée à la pensée de Heidegger ${ }^{18}$. L'expression du titre de sa contribution ( Se tenir dans le vrai») est empruntée à Heidegger (Einführung in die Metaphysik et Die Ursprung des Kunstwerkes) et indique en termes ontologiques ce que la notion de «savoir» exprime ontiquement. La vérité n'est pas de l'ordre de l'étant, mais de l'être lui-même, et elle exprime, en ce sens, le domaine de manifestation dans lequel tout étant (y compris nous-mêmes) se trouve et de ce fait est. L'auteur propose ensuite une «variante dans la pensée de l'être comme vérité ${ }^{19}$ ", qui consiste à relever que tout apparaître est en vérité le se soustraire de l'être lui-même, et que donc "disparition et manifestation ${ }^{20}$ " font un dans le phénomène de la vérité. Il retrouve une dernière fois Heidegger par la notion de Stimmung, qu'il interprète comme la possibilité d' « une approche émotionnelle du vrai ${ }^{21}$ ».

La deuxième section du recueil, intitulée «La question de la vérité dans les grandes religions ", réunit les contributions de Souleymane Bachir Diagne, Anna Ghiglione, Jacob Rogozinski et Danielle Cohen-Levinas. Bachir Diagne nous fait découvrir la figure d'un Descartes ante litteram: Abu Hamid Al-Ghazali (Algazel chez les latins; I058-I I I I) ${ }^{22}$. Le sectarisme religieux de l'époque d'Al-Ghazali jette, dans le propos de Bachir Diagne, une lumière sur notre présent multi-religieux. À travers une lecture aussi coranique, l'auteur fait d'un côté revivre une personnalité majeure de l'histoire de la pensée, et, de l'autre, montre que la vérité, certes à l'origine du pluralisme, ne demeure pas moins l'occasion d'une convergence. En nous déplaçant de l'autre côté de l'Asie, la spécialiste de la philosophie chinoise,

15. Pierre Gisel, «Résistances des particularités et pièges de l'universel. Pour un usage subversif des corps, des traditions et des frontières ", dans Jacques Ehrenfreud et Pierre Gisel, dir., Mises en scène de l'humain, Paris, Beauchesne, 2014, 266 pages.

16. Carla Canullo, «Religion et vérité: l'avenir d'une conjonction », dans Jean Grondin et Garth Green, dir., Religion et vérité. La philosophie de la religion à l'âge séculier, p. 69-76.

17. Ibid., p. 75 .

18. Yannick Courtel, «Se tenir dans le vrai ", dans Jean Grondin et Garth Green, dir., Religion et vérité. La philosophie de la religion à l'âge séculier, p. 77-85.

19. Ibid., p. 8I.

20. Ibid., p. 82.

21. Ibid.

22. Souleymane Bachir Diagne, «La "vraie" religion et le pluralisme», dans Jean Grondin et Garth Green, dir., Religion et vérité. La philosophie de la religion à l'âge séculier, p. 89-96. 
Ghiglione, présente de manière synthétique et efficace le statut de (non-) religion du confucianisme ${ }^{23}$. Elle réalise également un court excursus historique de la dynastie des Zhou à celle des Han, de Mao à nos jours. De thématique biblique, le très beau texte de Rogozinski traite du sens du sacrifice d'Abraham ${ }^{24}$. L'auteur engage un débat avec plusieurs traditions interprétatives (juives, chrétiennes et musulmanes) pour ensuite retrouver le Schelling de La philosophie de la révélation, qui, par l'interprétation des Noms de Dieu comme auto-révélation de Dieu lui-même, nous donne à comprendre le 'olah d'Abraham comme le passage de l'Idole au Nom, de Elohim à YHVH. Encore sur le monothéisme juif, cette fois lu à travers la voix de Levinas, la contribution de Cohen-Levinas ${ }^{25}$ est un commentaire de «Aimer la Torah plus que Dieu ${ }^{26}$ », texte ayant pour thème «la souffrance des innocents ", laquelle, en tant que "propre au peuple juif ${ }^{27}$ ", n'est plus l'objet d'une théodicée, mais apparaît comme la trace du passage de Dieu. Selon l'auteure, nous pourrions ainsi éclairer le sens de la mission immémoriale de ce peuple, fondée dans "la dimension prescriptive de la loi, au risque de l'athéisme» plutôt que dans «l'expérience du divin" ${ }^{28}$ ».

La troisième section intitulée "L'œuvre de Michel Henry et son apport à la question de la vérité de la religion» se compose des contributions de Garth Green, Jean Leclercq et Roberto Formisano. En soulignant que la relation entre la religion et la vérité est "l'intention ultime de L'essence de la manifestation" et également "la préoccupation constante de C'est moi la vérité ", Green cherche à montrer que, même si cette relation n'y est pas traitée de la même façon, les deux textes diffèrent surtout en ce qui concerne «la stratégie d'exposition des concepts fondamentaux de la théologie henryenne $^{29}$ ». Le texte de Leclercq dégage le statut de la vérité qui est propre au christianisme et qui tient ensemble, de façon unique en son genre dans l'œuvre henryenne, Vie et Ipséitée ${ }^{30}$. Fruit d'un minutieux travail archivis-

23. Anna Ghiglione, "Les vérités du confucianisme. Esquisse d'une "chorégraphie morale" ", dans Jean Grondin et Garth Green, dir., Religion et vérité. La philosophie de la religion à l'âge séculier, p. 97-103.

24. Jacob Rogozinski, "De l'Idole au Nom: deux visages de Dieu », dans Jean Grondin et Garth Green, dir., Religion et vérité. La philosophie de la religion à l'âge séculier, p. I05-I I 5.

25. Danielle Cohen-Levinas, "Religion sans vérité. Quelques réflexions sur le monothéisme juif chez Emmanuel Levinas ", dans Jean Grondin et Garth Green, dir., Religion et vérité. La philosophie de la religion à l'âge séculier, p. II7-I 25.

26. Emmanuel Levinas, "Aimer la Torah plus que Dieu», dans Difficile liberté: essais sur le judaïsme, Paris, Albin Michel, I963, 333 pages.

27. Danielle Cohen-Levinas, "Religion sans vérité. Quelques réflexions sur le monothéisme juif chez Emmanuel Levinas ", p. I 20.

28. Ibid., p. I23.

29. Garth Green, "Vérité et non-vérité dans la philosophie de la religion de Michel Henry ", dans Jean Grondin et Garth Green, dir., Religion et vérité. La philosophie de la religion à l'âge séculier, p. I29-I37.

30. Jean Leclercq, "Vie et méthode. Le tournant épistémologique de Michel Henry", dans Jean Grondin et Garth Green, dir., Religion et vérité. La philosophie de la religion à l'âge séculier, p. I39-I47. 
tique, l'apport de Formisano ${ }^{31}$ vise, à travers la reconstruction des lectures henryennes des décennies I930 et I940, à faire ressortir la genèse du problème de la religion chez le phénoménologue. On y retrouve aussi des observations en polémique avec Dominique Janicaud et son Tournant théologique de la phénoménologie française.

La quatrième section ( «Religion, art et alliance») rassemble les textes de Jean-Luis Vieillard-Baron, Philippe Capelle-Dumont, Marie-Anne Lescourret, Yasuhiko Sugimura, Jorge Luis Roggero. Vieillard-Baron illustre de manière savante la portée théologique de la peinture italienne et française, notamment à l'âge baroque ${ }^{32}$. La contribution de Capelle-Dumont ${ }^{33}$ porte sur la notion biblique d' "alliance» comme un véritable concept phénoménologique apte à rendre compte de "l'impossibilité facticielle de choisir entre ce qui toujours est là selon un geste de précédence et ce (lui) qui est là, affecté, bouleversé certes, mais qui, le nommant [...] forme décisivement un autre précédent ${ }^{34} »$. Lescourret fait le portrait de la vie intérieure d'un Wittgenstein «partisan d'une religion sans textes et sans Dieu ${ }^{35}$ ». Sugimura montre que la réception de Heidegger dans la phénoménologie française peut être pensée à partir de la notion de "témoignage ", alors que l' "autoéveil» (jikaku, et non pas jikoishiki, «conscience de soi») serait la marque de la lecture de Heidegger faite par l'École de $\mathrm{Kyoto}^{36}$. L'article de Sugimura contient également quelques notices historiques sur la pénétration de Heidegger dans la pensée japonaise. Après une présentation du rapport idole-icône dans l'œuvre de Jean-Luc Marion, Roggero se demande pour sa part s'il n'y a pas en dernière analyse au sein de la question de l'icône "une sorte d'imbrication entre philosophie et religion chez Marion ${ }^{37}$ ».

La cinquième section ("La vérité de la religion et la politique") recueille les interventions de Pierre Manent, Yves-Charles Zarka et Yann

31. Roberto Formisano, «Phénoménologie matérielle et philosophie de la religion. Relire Michel Henry à la lumière des inédits ", dans Jean Grondin et Garth Green, dir., Religion et vérité. La philosophie de la religion à l'âge séculier, p. I49-I 55.

32. Jean-Luis Vieillard-Baron, "L'expression artistique de la vérité religieuse à l'âge séculier ", dans Jean Grondin et Garth Green, dir., Religion et vérité. La philosophie de la religion à l'âge séculier, p. I 59-I68.

33. Philippe Capelle-Dumont, "Alliance et vérité», dans Jean Grondin et Garth Green, dir., Religion et vérité. La philosophie de la religion à l'âge séculier, p. I69-I76.

34. Ibid., p. I75.

35. Marie-Anne Lescourret, "Le religieux comme regain de l'intériorité chez Wittgenstein ", dans Jean Grondin et Garth Green, dir., Religion et vérité. La philosophie de la religion à l'âge séculier, p. I77-I 83 .

36. Yasuhiko Sugimura, "Témoignage et auto-éveil. Deux approches de l'expérience de vérité pour une philosophie de la religion "post-heideggérienne" ", dans Jean Grondin et Garth Green, dir., Religion et vérité. La philosophie de la religion à l'âge séculier, p. I85-I9I.

37. Jorge Luis Roggero, "La vérité de l'idole et de l'icône. Le rapport entre l'art et la religion chez Jean-Luc Marion ", dans Jean Grondin et Garth Green, dir., Religion et vérité. La philosophie de la religion à l'âge séculier, p. 193-200. 
Schmitt. Manent («Liberté et vérité. Remarques sur l'État moderne», p. 203-2I2) imagine une dialectique dramatique entre liberté et vérité, où la première s'imposerait aujourd'hui en Occident comme revendication radicale au détriment de la vérité ${ }^{38}$. La contribution de Zarka ${ }^{39}$ mobilise un penseur comme Yeshayahu Leibowitz pour rappeler que le «cercle de l'obsession et de l'occultation de la vérité » qui représente le dénominateur commun des religions dans le monde sécularisé, ne doit pas faire oublier les différences spécifiques propres à chacune d'elles. L'auteur renvoie à l'idée d'une « reconnaissance sans réconciliation » qu'il avait déjà développée dans Difficile tolérance. Grâce à une approche analytique du politique, Schmitt propose quant à lui de déterminer à l'intérieur de l'État laïc les différents espaces publics dans lesquels les «raisons religieuses» ne rivaliseraient pas avec les « raisons neutres» de la laïcité ${ }^{40}$.

La sixième et dernière section ( "Nouvelle perspectives») fait intervenir les jeunes voix de Joël Madore, Guillaume Saint-Laurent, Mathieu Scraire et Marc-Antoine Vallée. La contribution de Madore voit dans le « désenchantement du monde» moderne (Max Weber) un milieu paradoxalement fertile pour une foi religieuse renouvelée ${ }^{41}$. Le propos est rigoureux et la lecture de Kant profonde, mais ce qui fait défaut à sa contribution, c'est peut-être une confrontation avec Nietzsche. Saint-Laurent présente une intéressante lecture de Charles Taylor à la lumière du criticisme kantien ${ }^{42}$. La contribution de Scraire consiste à déterminer quelle est la valeur de la religion $^{43}$; l'auteur trouve la réponse dans l'obéissance, interprétée en termes ontologiques et cosmologiques comme "laisser s'imposer l'être». Vallée tâche quant à lui de réhabiliter le sens d'une vérité personnelle, face à l'impersonnalité des propositions scientifiques ${ }^{44}$. C'est à partir d'un horizon

38. Pierre Manent, «Liberté et vérité. Remarques sur l'État moderne», dans Jean Grondin et Garth Green, dir., Religion et vérité. La philosophie de la religion à l'âge séculier, p. 203$2 \mathrm{I} 2$.

39. Yves-Charles Zarka, "Obsession et occultation de la vérité dans les religions ", dans Jean Grondin et Garth Green, dir., Religion et vérité. La philosophie de la religion à l'âge séculier, p. 2 I I-22I.

40. Yann Schmitt, "La neutralité est-elle impossible aux croyants?", dans Jean Grondin et Garth Green, dir., Religion et vérité. La philosophie de la religion à l'âge séculier, p. 223229.

41. Joël Madore, «La foi dans le doute: interroger Marcel Gauchet à partir d'Emmanuel Kant", dans Jean Grondin et Garth Green, dir., Religion et vérité. La philosophie de la religion à l'âge séculier, p. 233-240.

42. Guillaume Saint-Laurent, «La religion dans les limites de la raison herméneutique. Sur la question de la vérité de la religion chez Charles Taylor », dans Jean Grondin et Garth Green, dir., Religion et vérité. La philosophie de la religion à l'âge séculier, p. $24 \mathrm{I}-258$.

43. Mathieu Scraire, "En quel sens parler de la "valeur de la religion" ? , dans Jean Grondin et Garth Green, dir., Religion et vérité. La philosophie de la religion à l'âge séculier, p. $25 \mathrm{I}-258$.

44. Marc-Antoine Vallée, "La vérité en personne(s)», dans Jean Grondin et Garth Green, dir., Religion et vérité. La philosophie de la religion à l'âge séculier, p. 259-265. 
chrétien que la question est abordée, et c'est chez Bonaventure que l'auteur retrouve les outils conceptuels qui conviennent à l'expérience qu'il cherche à décrire: la «nihilitét5 ${ }^{45}$ et l'humilité.

L'ouvrage se conclut par un texte écrit par Garth Green ${ }^{46}$, qui laisse l'ouvrage en héritage aux lecteurs, "car la signification d'un événement et celle d'un texte n'appartiennent qu'à l'avenir auquel ils donnent lieu ${ }^{47}$ ».

Religion et vérité est d'abord un ouvrage courageux, en ce qu'il tente de montrer, à travers un spectre très large d'approches, l'« incontournabilité» (pour le dire avec Heidegger) de la vérité religieuse. C'est pourquoi il s'agit d'un livre susceptible d'intéresser tant les spécialistes de philosophie de la religion, que les lecteurs prêts à se questionner sur le sens et le fondement philosophique de leur croyance ou non-croyance. Certaines positions exprimées là ne manqueront pas de contrarier certains, mais même les thèses les plus audacieuses laissent transparaître en filigrane une réflexion philosophique. Finalement, on remarque que l'ouvrage est dédié «à la mémoire des victimes des attentats du 7 janvier et du I3 novembre 20I5»: puisqu'il s'agit des actes d'un colloque ayant eu lieu au Québec, rien n'aurait empêché de dédier (aussi) cette belle publication à la mémoire des victimes de l'attentat du 29 janvier 20I7 à la Grand Mosquée de Québec. Peut-être le délai éditorial ne l'a-t-il pas permis?

GIULIO MELLANA

Université Laval, Québec Sorbonne Université, Paris

\section{Céline Spector, Éloges de l'injustice. Philosophie face à la déraison, Paris, Seuil, 2016, 240 pages.}

«Avions-nous oublié le mal?»: cette question — reprise du titre de l'ouvrage de Jean-Pierre Dupuy — est au cœur du livre de Céline Spector, Éloges de l'injustice, la philosophie face à la déraison. Ainsi, si le livre de JeanPierre Dupuy, paru en septembre 2002, interrogeait l'incapacité de la philosophie politique contemporaine à comprendre les attentats du I I septembre 200I, l'ouvrage de C. Spector s'ouvre — et se clôt — sur la difficulté du discours philosophique à produire une analyse convaincante des attentats qui, particulièrement depuis 2015, ont touché la France. On pourrait croire de la sorte que l'ouvrage répond à l'urgence de l'événement - avec les risques que comporte cette forme d'écriture. Mais il n'en est rien. En effet,

45. Ibid., p. 262.

46. Garth Green, « Religion et vérité: le passé et le futur d'une conjonction », Jean Grondin et Garth Green, dir., Religion et vérité. La philosophie de la religion à l'âge séculier, p. 26727 I.

47. Ibid., p. 269. 